\title{
Emploi d'un vaccin antibovipestique produit sur cultures cellulaires dans la prophylaxie de la peste des petits ruminants au Dahomey
}

\author{
NOTE PRELIMINAIRE
}

\author{
par P. BOURDIN, M. RIOCHE, A. LAURENT
}

\begin{abstract}
RESUME
La peste des petits ruminants (P. P. R.) provoquant des pertes croissantes chez les moutons et les chèvres de certains Etats d'Afrique de l'Ouest, des recherches ont été entreprises en 1969 afin de mettre au point une méthode de prophylaxie médicale efficace. Après un bref rappel concernant la maladie et les travaux s'y rapportant, les auteurs rapportent leurs expérimentations sur l'emploi et l'efficacité du vaccin contre la peste bovine produit sur cultures cellulaires dans l'immunisation des petits ruminants contre la P.P.R. Ce vaccin a été choisi en raison des relations antigéniques étroites existant entre le virus P.P.R. et le virus P.B. et de sa préparation aisée.

Si les résultats des essais expérimentaux peuvent être discutés, ceux des vaccinations effectuées « sur le terrain » dans les conditions naturelles d'élevage sont excellents et l'immunité dure au moins un an. L'appréciation de l'immunité naturelle et acquise par la méthode cinétique de titrages des anticorps neutralisant le virus de la peste bovine pose des problèmes dont l'étude fait actuellement l'objet de recherches complémentaires.
\end{abstract}

La peste des petits ruminants ou P.P.R. existe à l'état endémique au Dahomey. En 1966, le Gouvernement de ce pays s'est inquiété de l'augmentation constante des pertes et a demandé au Gouvernement français de financer les recherches visant à la mise au point d'une prophylaxie médicale efficace. Ces recherches ont débuté en janvier 1969, et un an après les premiers essais, il est possible de donner quelques résultats intéressants sur la valeur du vaccin utilisé. Auparavant, il sera fait un bref rappel des principaux caractères de la maladie.

\section{A. RAPPEL SOMMAIRE SUR LA P.P.R.}

\section{Epidémiologie :}

L'étude épidémiologique révèle que la P.P.R. est en progrès constants au Dahomey. Entre 1953 et 1968 , le nombre des foyers a augmenté de 2 à 230 , celui des malades de 180 à 4.200 et les morts de 18 à 4.200 ; ces chiffres proviennent des archives du Service de l'Elevage du Dahomey. Une enquête faite en 1969 dans le cadre de la convention révèle que ces évaluations sont très en-dessous de la réalité. 
Du point de vue géographique, la maladie sévit surtout dans le Sud et le Centre du pays; elle est rarement observée dans le Nord. La P.P.R. existe au Togo, au Nigéria et en Côte d'Ivoire. Au Sénégal, il y a eu deux grandes épizooties en 1956 et 1962.

$\mathrm{Au}$ Dahomey, la race joue un rôle important : en effet, les chèvres naines, dites de race lagunaire sont beaucoup plus sensibles que les chèvres sahéliennes d'un format plus grand.

\section{Symptomatologie}

La P.P.R. atteint uniquement les petits ruminants, les chèvres sont plus sensibles que les moutons. Après 3 à 4 jours d'incubation apparaît la fièvre qui peut atteindre $42^{\circ} \mathrm{C}$, rapidement accompagnée de jetage muqueux, pu1s purulent. A partir du $6^{\circ}$ jour, les gencives s'ulcèrent. Une diarrhée suit habituellement, provoquant une déshydratation rapide des malades qui ne mangent plus, boivent beaucoup et meurent dans un état de profonde prostration. (MORNET, ORUE et collab. 1955).

Les complications les plus fréquentes sont soit d'origine bactérienne (pneumonie ou bronchopneumonie à partir desquelles on isole des pasteurelles ou des mycoplasmes), soit d'origine parasitaire (réveil de coccidioses intestinales latentes). Le plus souvent les femelles gestantes avortent.

\section{Etiologie}

MORNET, ORUE et collab. (1955) ont montré que la P.P.R. est due à un virus. Ses propriétés physiques, chimiques et antigéniques sont pour la plupart identiques à celles du virus de la peste bovine (GILBERT et MONNIER, 1962) (BOURDIN et LAURENT, 1967) (LAURENT, 1968). Comme celui-ci, il peut être classé dans la famille des Paramyxoviridae, sous-groupe M.R.D. (Measles, Rinderpest, Distemper).

\section{Traitement}

Il n'y a pas de traitement spécifique de la P.P.R.; cependant, l'administration de produits actifs contre les complications microbiennes ou parasitaires (antibiotiques, sulfadimarazine, phénothiazine) peut abaisser le taux de mortalité. Leur emploi est malheureusement trop onéreux.

\section{Prophylaxie médicale}

CATHOU (1947-48-49-51) fait des essais de séro-protection sur des animaux sains ou malades avec du sérum de bovins hyperimmunisés contre la peste bovine. Il obtient des résultats encourageants mais le prix de revient de cette méthode est très élevé.

Des essais de vaccination ont également été faits, d'une part avec un vaccin antibovipestique inactivé (GARGADENNEC et LALAN$\mathrm{NE}, 1942$ ), d'autre part avec le vaccin antibovipestique lapinisé (MORNET, ORUE et collab. 1956). Les résultats ne sont pas concluants.

GILBERT et MONNIER en 1962, réussissent à modifier le virus P.P.R. par 51 passages sur cultures de cellules de reins d'embryon de mouton. Ce virus dépourvu de pouvoir pathogène pour les petits ruminants leur confère une immunité suffisante mais le nombre des résultats expérimentaux est trop réduit pour être significatif. Nos récents essais d'immunisation à l'aide de ce vaccin ont été décevants.

\section{B. ETUDE DE L'EFFICACITE DE L'IMMUNISATION DES PETITS RUMINANTS CONTRE LA P.P.R. PAR LE VACCIN ANTIBOVIPESTIQUE}

En raison des résultats obtenus avec le vaccin anti-P.P.R. de GILBERT et MONNIER (1962) et nous basant sur l'étroite parenté antigénique des virus P.P.R. et P.B. nous avons songé à tester le pouvoir protecteur du vaccin antibovipestique vivant modifié, contre la P.P.R. Ce vaccin largement utilisé dans la prophylaxie de la peste bovine est préparé sur cellules rénales d'embryon de veau inoculées avec le $60^{\circ}$ passage de la souche RPKO/BK isolée et mise au point par PLOWRIGHT et FERRIS (1962).

\section{MATERIEL ET METHODES}

Les essais du vaccin ont été faits ainsi :

- Vaccination de lots expérimentaux constitués par des animaux achetés sur les marchés, enfermés et soumis à une surveillance constante. 
- Vaccination sur le terrain des animaux de tout un village ou de tout un canton avec possibilité pour l'éleveur d'accepter ou de refuser. Ce procédé a l'avantage de laisser les animaux dans leur milieu naturel, de multiplier les vaccinations et permet aux utilisateurs d'apprécier les résultats de cette opération.

\section{Vaccination de lots expérimentaux}

Ces essais sont faits conjointement au Dahomey, pays infecté, et au Sénégal où la P.P.R. n'est actuellement pas signalée.

\section{Animaux}

La plupart des expériences sont faites sur des caprins, espèce plus sensible à la maladie naturelle. Au Dahomey, les chèvres sont achetées sur les marchés après un examien clinique qui ne permet malheureusement pas d'éliminer les animaux contaminés ou en incubation.

Enfermés dans des parcs, les animaux reçoivent une alimentation à base de manioc, maïs, tourteau d'arachide et coquilles d'huîtres, distribuée sous la forme de provende. Dans la mesure du possible, ils reçoivent également un peu de verdure. Ce mode de vie est très différent de celui auquel ils sont habitués et les met en état de moindre résistance.

Au Sénégal, les animaux sont achetés directement chez les éleveurs. Mis dans des bergeries, ils reçoivent une nourriture variée, proche de celle reçue habituellement. La maladie n'étant pas signalée depuis 1962 , la vaccination a toutes les chances d'être faite en milieu sain.

\section{Contrôle sérologique}

Les résultats exposés ici intéressent uniquement les chèvres. Les sérums des animaux sont testés avant et après vaccination par la méthode de séroneutralisation cinétique quantitative adaptée au virus de la peste bovine par BOURDIN et BERNARD (1967) et RIOCHE (1968-1969) : le mélange virus-sérum est laissé une heure à $37^{\circ} \mathrm{C}$ dans des tubes à hémolyse puis on ajoute une quantité fixe de cellules sensibles en suspension dans un milieu nutritif; le tout est ensuite recouvert d'une couche d'huile de paraffine et laissé à $37^{\circ} \mathrm{C}$. Les cellules utilisées dans ce test sont issues de la lignée cellulaire MDBKC établie par MADIN et DARBY (1968) à partir d'un rein de bovin adulte.
En raison de l'étroite parenté antigénique existant entre les virus P.B. et P.P.R., on pouvait penser a priori que la présence d'anticorps neutralisant le virus P.B. dans le sérum des petits ruminants était corollaire d'une solide résistance à la P.P.R., aussi bien dans le cas d'une maladie naturelle que dans celui d'une inoculation expérimentale. Les recherches faites au Dahomey puis au Sénégal ont montré que cette hypothèse ne se vérifiait pas chez tous les animaux. Aussi à l'heure actuelle tous les sérums prélevés en 1969 au Dahomey et au Sénégal sont-ils l'objet d'une double séroneutralisation. Pour chaque sérum on titre les anticorps neutralisants en présence de virus P.B. et également du virus P.P.R. Pour ce dernier la souche utilisée a été adaptée auparavant à la lignée cellulaire MDBKC.

\section{Vaccin}

Il est constitué par le vaccin antibovipestique de cultures cellulaires mis au point par PLOWRIGHT et FERRIS (1962) et préparé selon les recommandations de JOHNSON (1962). Conservé à l'état lyophilisé, il est mis en suspension au moment de l'emploi dans $50 \mathrm{ml}$ d'eau distillée refroidie. La dose habituellement injectée est de $1 \mathrm{ml}$; elle correspond à environ 5.000 DI 50 CT. Au Dahomey, au cours d'un essai de titrage du vaccin fait sur des dilutions décimales croissantes, les animaux ont reçu environ $10.000,1.000,100,10$ et 1 DI $50 \mathrm{CT}$ selon les lots.

\section{Epreuve des animaux et des témoins}

L'inoculum d'épreuve est unc souche de virus P.P.R. isolée au Dahomey en janvier 1969, imnatriculée 45 G. Après deux passages sur culture cellulaire de reins d'embryon de mouton. son pouvoir pathogène a été confirmé par inoculation à des chèvres sensibles. La suspension virulente lyophilisée est reconstituée au moment de l'emploi; son titre est de $10^{5,2}$ DICT $50 / \mathrm{ml}$ et chaque animal reçoit 500 DICT 50.

\section{RESULTATS}

\section{Vaccination et épreuve des lots expérimentaux}

a) Au Dahomey 


\section{- Première partie}

80 chèvres sont achetées sur le marché et 76 vaccinées dans un délai de 2 à 5 jours; 4 sont conservées comme témoins. Entre le $3^{\circ}$ et le $16^{\mathrm{e}}$ jour suivant la vaccination, 57 animaux meurent de P.P.R., à savoir les 4 témoins et 53 vaccinés. Passé ce temps, les mortalités cessent.

Parmi les chèvres vaccinées mortes de P.P.R. :

- 30 ont reçu de 1 à 100 D1 50 CT de virus vaccinal (la mortalité dans ce lot est supérieure à 80 p. 100);

- 46 ont reçu de 1.000 à 10.000 DI 50 CT de virus vaccinal (le taux de mortalité dans ce lot est de 50 p. 100).

33 moutons sont achetés et vaccinés dans les mêmes conditions, 7 animaux seulement meurent de P.P.R. entre le $1^{\mathrm{cr}}$ et le $16^{\circ}$ jour suivant la vaccination.

\section{- Deuxième partie}

L'état de résistance des animaux survivants pouvant être attribué aussi bien à la maladie naturelle qu'à la vaccination, un deuxième essai est fait sur un petit nombre de chèvres acquis 1 mois après le dernier cas de mortalité observé dans le premier lot. 4 chèvres sont achetées, saignées pour le contrôle sérologique et immédiatement vaccinées avec 5.000 DI $50 \mathrm{CT}$ de vaccin tissulaire.

Epreuve des chèvres et des moutons. Elle est faite sur les animaux suivants :

- 23 chèvres et 26 moutons vaccinés 60 jours auparavant et ayant résisté à la maladie naturelle;

- 4 chèvres vaccinées 15 jours avant;

- 5 témoins acquis le mềme jour.

Tous les animaux vaccinés résistent. Trois témoins sur 5 meurent de P.P.R.

On doit préciser que la première partie de cette expérimentation a été faite dans des conditions extrêmement défavorables pour les animaux. En effet, l'introduction de sujets en incubation ou contaminés, le stress physiologique dû à la claustration et à la nourriture mal adaptée favorisent l'apparition d'une épizootie. En fait, le vaccin a été testé dans un foyer et sur des animaux placés dans de mauvaises conditions d'hygiène et d'alimentation.
On peut simplement retenir que dans ce premier lot la résistance à la maladie naturelle n'a été acquise que 16 jours après le début de la vaccination puisque c'est à ce moment que les mortalités dues à la P.P.R. ont cessé.

La vaccination faite sur le deuxième lot malheureusement réduit à 4 chèvres indique que malgré leur grande sensibilité à la P.P.R., les chèvres résistent à l'inoculation d'épreuve 15 jours après l'injection vaccinale.

\section{b) Au Sênégal}

Les essais sont repris sur 40 chèvres qui reçoivent de 5.000 à 10.000 DI $50 \mathrm{CT}$ de virus vaccinal. Ces animaux sont conservés 1 mois et pendant ce délai, 4 sujets meurent avec des syndromes pulmonaires. L'examen nécropsique montre des lésions de pneumonie à partir desquelles le service de bactériologie isole plusieurs mycoplasmes et plus rarement des pasteurelles.

L'épreuve est faite sur 36 chèvres restantes 1 mois après la vaccination. Durant les 4 premiers jours, 4 chèvres meurent de pneumonie, puis entre le $8^{\mathrm{e}}$ et le $12^{\mathrm{e}}$ jour, deux autres meurent de P.P.R. Les 30 animaux restants résistent.

\section{Vaccination sur le terrain}

Un relevé récent reçu du Dahomey précise que le nombre d'animaux vaccinés par les agents du Service de l'Elevage dépasse 20.000 . Tous les utilisateurs reconnaissent l'efficacité du vaccin.

Dans la région du Mono, située au sud-ouest du Dahomey, habituellement très touchée par la P.P.R., le chef de région indique que ses agents ont vacciné 11.600 petits ruminants. Dans ses commentaires, il déclare: «Tous les animaux vaccinés ont résisté à une nouvelle flambée de la maladie. Les paysans qui, par méfiance ou par négligence, n'ont pas présenté leurs animaux à la vaccination ont vu leur troupeau littéralement anéanti. " Il ajoute que même dans des foyers où ses agents sont intervenus, la mortalité a cessé brusquement une semaine après la vaccination.

\section{Etude sérologique}

Ce travail fera l'objet d'un article complet par la suite. 
En résumé, les titrages faits par la méthode cinétique basée sur la recherche des anticorps neutralisant le virus P.B. sur les sérums prélevés avant et après vaccination montrent que l'utilisation de la souche vaccinale de PLOWRIGHT et FERRIś (1962), utilisée habituellement dans la prophylaxie de la peste bovine, provoque la formation d'anticorps neutralisants ou en augmente le titre chez les petits ruminants qui en possédaient auparavant. En effet, avant la vaccination 32,8 p. 100 des sérums ont des anticorps et 68,2 p. 100 en sont dépourvus; après la vaccination, tous les animaux ont des anticorps. Les titrages sérologiques faits les $8^{\mathrm{e}}, 14^{\mathrm{e}}, 21^{\mathrm{e}}$ et $30^{\mathrm{e}}$ jour qui ont suivi la vaccination montrent que le titre en anticorps augmente régulièrement à partir du $14^{\mathrm{e}}$ jour pour atteindre un maximum vers le $30^{\circ}$ jour.

En ce qui concerne la détermination précise du titre en anticorps neutralisants, à partir duquel une chèvre peut être considérée comme immunisée, la méthode utilisée ne permet pas de le fixer avec précision. En effet, quand on compare les titrages faits sur des sérums provenant d'animaux vaccinés et ceux faits sur des sérums d'animaux non vaccinés, on constate :

- dans le cas des chèvres vaccinées que: les sujets possédant des anticorps au $1 / 20$ ou au $1 / 40$ résistent parfaitement à l'inoculation expérimentale;

- dans le cas des chèvres non vaccinées, les sujets possédant des anticorps au $1 / 20$ sont sensibles dans 80 p. 100 des cas à la maladie naturelle et dans 50 p. 100 des cas pour celles dont le titre est égal à $1 / 40$.

Comme cela a été dit auparavant tous ces sérums sont l'objet actuellement d'un double titrage en présence des virus P.B. et P.P.R. afin de vérifier si les mêmes résultats sont observés en présence de ce second virus.

\section{DISCUSSION ET CONCLUSION}

La P.P.R. sévit régulièrement dans plusieurs pays de l'Ouest africain et les pertes qui en résultent sont en augmentation constante dans certains états.

Les moyens de lutte mis en œuvre jusqu'à présent sont peu efficaces ou trop chers pour être généralisés. Les recherches entreprises au Dahomey et au Sénégal avaient pour principal objectif de vérifier la valeur immunitaire du vaccin préparé à partir de la souche RP KO/BK de PLOWRIGHT et FERRIS (1962) modifiée par 60 passages sur cellules rénales d'embryon de veau et qui est très largement employée pour la protection des bovins contre la peste bovine.

Les résultats obtenus au cours des expériences faites au Dahomey montrent que l'efficacité du vaccin sur des animaux maintenus en claustration est parfois aléatoire en raison des difficultés à obtenir des chèvres non contaminées et à les habituer ensuite à un mode de vie très différent du mode habituel.

Au Sénégal où la maladie n'était pas signalée au moment de l'expérimentation, en 1969, il a été plus facile de vérifier l'efficacité du vaccin à condition de ne pas conserver les animaux trop longtemps dans les bergeries pour éviter les affections pulmonaires le plus souvent dues à des mycoplasmes.

Des recherches complémentaires actuellement en cours permettront de fixer avec précision le moment à partir duquel les animaux résistent à l'inoculation expérimentale.

Les résultats obtenus au cours des vaccinations massives qui ont été faites par les agents du Service de l'Elevage au Dahomey sont beaucoup plus démonstratifs. A la lecture des renseignements reçus et après une enquête très récente effectuée par l'un de nous sur place, il ressort que la maladie a disparu des localités où la vaccination a été faite sur tous les animaux en un seul temps. Les quelques épreuves qui ont pu être faites sur des sujets vaccinés depuis un an ont révélé qu'ils étaient encore protégés. En outre de nombreux échantillons de sérums prélevés sur des animaux vaccinés à des intervalles de temps variables sont en cours d'examen.

Tout récemment au Sénégal, la vaccination a prouvé son efficacité dans deux foyers apparus l'un en janvier, l'autre en mars 1970. Une enquête effectuée en avril dans le dernier foyer a permis de constater la disparition de la P.P.R. alors qu'elle continue à sévir dans un autre foyer situé à une vingtaine de kilomètres. En raison de la facilité d'accès des lieux, des contrôles pourront être faits l'année prochaine. 


\section{SUMMARY}

\section{A tissue culture vaccine against pseudorinderpest of goats - Fields experiments in Dahomey}

The pest of sheep and goats (P.P.R.) causing crescent losses in some countries of West Africa, new works have been carried on in 1969 in order to develop an efficient method of immunization. After a brief description of this disease and an account of past references, the authors describe their experiments on use and efficiency of a rinderpest cell culture vaccine for protection of sheep and goats against P.P.R. This vaccine was selected because the closed antigenic relationship between P.P.R. and rinderpest viruses have been demonstrated and the preparation is an easy one. If the results of experimental trials in laboratory can be discussed, the vaccination in the field, in natural conditions, entails a valuable immunity that lasts at least one year. The study of natural and artificial immunity by the titration of neutralizing antibodies constitutes an unsolved problem which is going to be studied thanks to further immunological investigations.

\section{RESUMEN}

Empleo de una vacuna antibovipestica producida sobre cultivos celulares en la profilaxia de la peste de los pequeños rumiantes en Dahomey. Nota preliminar

La peste de los pequeños rumiantes (P.P.R.) provocando cada vez más perdidas en las ovejas y cabras de c1ertos Estados de Africa del Oeste, se han emprendido en 1969 investigaciones para establecer un método de profilaxia medical eficaz. Después de una breve descripción de la enfermedad y una revista de los trabajos ya efectuados, los autores notan sus experimentaciones sobre el empleo y la eficacia de la vacuna contra la peste bovina producida sobre cultivos celulares en la inmunización de los pequeños rumiantes contra la P.P.R. Se elegió dicha vacuna a causa de las relaciones antigenicas estrechas existiendo entre el virus P.P.R. y el virus P.B. y de su preparación fácil. Si se pueden discutir los ensayos experimentales, son excelentes los de las vacunaciones efectuadas «sobre el terreno * en las condiciones naturales de cria y la inmunidad permanece por lo menos durante un año. La apreciación de la inmunidad natural y adquirida por el método cinetico de dosajes de los anticuerpos neutralizando el virus de la peste bovina plantea problemas que se estudian actualmente.

\section{BIBLIOGRAPHIE}

BOURDJN (P.), BERNARD (G.), «Application de la méthode de séroneutralisation cinétique à la recherche des anticorps neutralisant le virus de la peste bovine chez les bovins, les caprins et les ovins ", Rev. Elev. Méd. vét. Pays trop., 1967, 20, 4, 531-535.

BOURDIN (P.), LAURENT (A.), «Note sur la structure du virus de la peste des petits ruminants ". Rev. Elev. Méd. vét. Pays trop., 1967, 20, 3, 383-386.

CATHOU (P.), "Rapport annuel du Service de l'Elevage au Dahomey. 1941. 1942, 1948, 1949, 1951 »,

GARGADENNEC (L), LALANNE (A.), «La peste des petits ruminants", Bull. Serv. Zoot. A.O.F., $1942, \mathbf{5}, 16$.

GILBERT (Y.), MONNIER (J.), «Adaptation du virus de la P.P.R. aux cultures cellulaires, Rev. Elev. Méd. vét. Pays trop., 1962, 15, 4, 321-335.

JOHNSON (R. H.), "Rinderpest in tissue culture. I. Methods for virus production », Brit. vet. J., 1962, 118, 107-116.

LAURENT (A.), «Aspects biologiques de la multiplication du virus de la peste des petits rumi- nants sur les cultures cellulaires", Rev. Elev. Méd vét. Pays trop., 1968, 21, 3, 297-308.

MADIN (S. H.), DARBY (N.D.), "Established kidney cell lines of normal adult bovin and ovin origin », Proc. soc. exp. Biol. Med., 1958, 98, 574-576.

MORNET (P.), ORUE (J.), GILBERT (Y.), THIERY (G.), SAW MAMADOU, «La peste des petits ruminants en Afrique occidentale française. Ses rapports avec la peste bovine ", Rev. Elev. Méd. vét. Pays trop., 1956, 9, 4, 313-342.

PLOWRIGHT (W.), FERRIS (R.D.), « Studies with rinderpest virus in tissue culture. The use of attenuated culture virus as a vaccine for cattle ", Res. Vet. Sci., 1962, 8, 172-182.

RIOCHE (M.), « Rapport 1968 sur le fonctionnement du Laboratoire National de l'Elevage et de Recherches vétérinaires de Dakar-Hann ».

RIOCHE (M.), « Adaptation en microtest de la technique de séroneutralisation cinétique pour la recherche et le titrage des anticorps neutralisant le virus de la peste bovine ", Rev. Elev. Méd. vét. Pays trop., 1969, 22, 4, 465-471. 